Draft Version SEPtember 28, 2018

Preprint typeset using $\mathrm{IAT}_{\mathrm{E}} \mathrm{X}$ style emulateapj v. 5/2/11

\title{
ON THE RECOVERY OF LOCAL GROUP MOTION FROM GALAXY REDSHIFT SURVEYS
}

\author{
Adi Nusser \\ Physics Department and the Asher Space Science Institute-Technion, Haifa 32000, Israel \\ e-mail: adi@physics.technion.ac.il \\ MARC DAVIS \\ Departments of Astronomy \& Physics, University of California, Berkeley, CA. 94720 \\ ENZO BRANCHINI \\ Department of Physics, Università Roma Tre, Via della Vasca Navale 84, 00146, Rome, Italy \\ INFN Sezione di Roma Tre, Via della Vasca Navale 84, 00146, Rome, Italy \\ INAF, Osservatorio Astronomico di Roma, Monte Porzio Catone, Italy \\ Draft version September 28, 2018
}

\begin{abstract}
There is a $\sim 150 \mathrm{~km} \mathrm{~s}^{-1}$ discrepancy between the measured motion of the Local Group of galaxies (LG) with respect to the CMB and the linear theory prediction based on the gravitational force field of the large scale structure in full-sky redshift surveys. We perform a variety of tests which show that the LG motion cannot be recovered to better than $150-200 \mathrm{~km} \mathrm{~s}^{-1}$ in amplitude and within a $\approx 10^{\circ}$ in direction. The tests rely on catalogs of mock galaxies identified in the Millennium simulation using semi-analytic galaxy formation models. We compare these results to the $K_{s}=11.75$ Two-Mass Galaxy Redshift Survey, which provides the deepest, widest and most complete spatial distribution of galaxies available so far. In our analysis we use a new, concise relation for deriving the LG motion and bulk flow from the true distribution of galaxies in redshift space.

Our results show that the main source of uncertainty is the small effective depth of surveys like the $2 \mathrm{MRS}$ that prevents a proper sampling of the large scale structure beyond $\sim 100 h^{-1} \mathrm{Mpc}$. Deeper redshift surveys are needed to reach the "convergence scale" of $\approx 250 h^{-1} \mathrm{Mpc}$ in a $\Lambda \mathrm{CDM}$ universe. Deeper survey would also mitigate the impact of the "Kaiser rocket" which, in a survey like 2MRS, remains a significant source of uncertainty. Thanks to the quiet and moderate density environment of the LG, purely dynamical uncertainties of the linear predictions are subdominant at the level of $\sim 90 \mathrm{~km} \mathrm{~s}^{-1}$. Finally, we show that deviations from linear galaxy biasing and shot noise errors provide a minor contribution to the total error budget.

Subject headings: Cosmology: theory, observations, large scale structure of the universe, dark matter
\end{abstract}

\section{INTRODUCTION}

The group of galaxies containing M31, the Milky Way (MW) and about a dozen other, much smaller, galaxies (excluding satellites) within $\sim 1.4 \mathrm{Mpc}$ form a bound system which is detached from the general cosmic expansion (Yahil et al. 1977). This Local Group (LG) of galaxies resides in mildly over-dense region characterized by a remarkably small velocity shear. Just like any other cosmological object, the LG is expected to move with a non-vanishing velocity relative to the general expanding background. The best approximation to the frame of reference defined by the cosmological background is undoubtedly based on temperature maps of the Cosmic Microwave Background (CMB). The high degree of dipole anisotropy in the temperature of the CMB on the sky (Kogut et al. 1993; Fixsen et al. 1996; Hinshaw et al. 2009 ) is interpreted as a Doppler boosting resulting from our motion through a highly isotropic thermal CMB photons. This interpretation has recently been reinforced by the detection of the corresponding modulation and aberration of the CMB fluctuations observed by the Planck

adi@physics.technion.ac.il máavis@̄berkeley.edu maavis@iderkeley.edu satellite (Planck Collaboration et al. 2013). The observed dipole yields a very precise measurement of the Solar barycenter velocity relative to the "CMB frame" of reference in which an observer at rest would measure a vanishing dipole anisotropy. Augmented with astronomical estimation of the LG motion relative to the Sun (c.f. $\$ 2$, the CMB dipole provides $V_{\mathrm{lg}}=627 \pm 22 \mathrm{~km} \mathrm{~s}^{-1}$ toward $(l, b)=\left(276^{\circ} \pm 3^{\circ}, 30^{\circ} \pm 3^{\circ}\right)$ as the reference value for the LG motion relative to the CMB frame (Kogut et al. 1993). In the standard cosmological paradigm (Peebles 1980), the LG is accelerated by the cumulative gravitational pull of the surrounding large scale structure. In linear theory, the peculiar velocity is proportional to the peculiar gravitational force field times the Hubble time, with proportionality factors depending on the background mass density. Therefore, it is natural to ask whether the observed large scale structure, as traced by the galaxy distribution, could indeed account for the LG motion.

This issue was recently studied by Davis et al. (2011) who found good agreement between the local velocity and gravitational fields, in contrast to the earlier data with an inferior velocity field, which gave irreconcilable differences between the two (Davis et al. (1996)). The 
earlier studies of (Yahil et al. 1980; Davis \& Huchra 1982 ) addressed the gravity versus velocity fields, with somewhat contradictory results, using either the angular positions and fluxes from photometric galaxy catalogs (Yahil et al. 1986; Meiksin \& Davis 1986; Harmon et al.|1987; Villumsen \& Strauss 1987; Plionis 1988; Bilicki et al. 2011) or the full 3D distribution of different types of extragalactic objects ranging from the infra-red selected galaxies of the IRAS 1.2 Jy catalog (Strauss et al. 1992 Webster et al. 1997; Zaroubi et al. 1999) and extension to fainter fluxes, the PSC $z$ catalog (Schmoldt et al. 1999a b, Rowan-Robinson et al. 2000; Basilakos \& Plionis 2006), optically selected galaxies (Lahav 1987; LyndenBell et al. 1989, Hudson 1993), mixed catalogs of infrared and optical galaxies (Lahav et al. 1988; D'Mellow et al. 2004), galaxy clusters selected from optical plates (Plionis \& Valdarnini 1991; Scaramella et al. 1991, 1994; Branchini et al. 1996 Dale et al. 1999) and, finally, Xray selected galaxy clusters (Plionis \& Kolokotronis 1998 Kocevski \& Ebeling 2006).

In this work we focus on determining how well the LG motion can be recovered from the observed galaxy distribution via linear instability theory. Detection of significant departures from theoretical expectations require a complete understanding and characterization of all possible error sources. In the case of cosmological dipoles these are (Schmoldt et al. 1999a)

- Cosmic variance from finite volume sampling. All sky galaxy surveys become significantly more dilute at larger distances, limiting the depth within which density fluctuations can be reliably probed for the reconstruction of the LG motion.

- Shot noise from sparse sampling of the mass tracers.

- Highly non-linear dynamical effects that spoil the relation the tight relation between the gravity and the LG motion. They include nonlinear motions and growth of density fluctuations.

- Deviations from strict linear biasing between galaxies and the underlying mass density fluctuations.

- Observational uncertainties and biases arising from incompleteness and selection effects in the parent objects' catalogue.

The most appropriate route to estimate the impact of these uncertainties and assess the adequacy of linear theory has been pioneered by Davis et al. (1991). It relies on the extensive use of realistic mock galaxy catalogs extracted from N-body simulation, since they simultaneously account for non nonlinear effects as well as selection effects specific to the specific dataset. In particular, the mocks should have LG candidates residing in a mild density region and small velocity shear, as in the real data. Although, one can attempt to account for non-linear dynamical effects within a likelihood formalism by quantifying the "decoherence" between the gravity and the peculiar velocity fields (Chodorowski \& Ciecielag 2002. Ciecielg \& Chodorowski 2004; Chodorowski et al. 2008), we opt here to rely on mock catalogs extracted from fully nonlinear $N$-body simulation. While we aim at a general discussion of the problem, we shall consider here the case of the Two-Mass Redshift Survey (2MRS) (Huchra et al. 2012 ), i.e. the the deepest nearly-all sky survey of angular positions and spectroscopic galaxy redshifts limited to $K_{s}=11.75$ and arguably the best sample of objects to estimate the LG motion.

The outline of the paper is as follows. A brief description of how the LG motion has been measured in the literature is given in $\$ 2$. In $\$ 3$, we review the linear theory predictions and offer a new useful relation for deriving the LG motion from a given distribution of mass tracers in redshift space. In 4 we first test linear theory as measured by the full dark matter distribution and the full volume-limited galaxy distribution. We characterize the biasing relation of the galaxy catalogs, address the reliability of linear reconstruction of the LG motion, and assess the impact shot noise errors. In $\$ 5.1$ we consider the analysis when applied to mock $2 \mathrm{RMS}$ catalogs. The results are presented in $\$ 5.2$ in which we compare the LG motions obtained from the distribution of mock galaxies to that measured directly in the N-body simulation. The impact of the so-called Kaiser effect is outlined in a separate 86 and, finally, we end with a general discussion in 87. In the Appendix the reader will find a detailed derivations of the linear theory relations used here and farther discuss the dependence on the so-called distortion parameter $\beta$ of peculiar velocities reconstructed from redshift space data.

\section{THE LOCAL GROUP}

The identification of the LG of galaxies and determining its motion in the heliocentric and CMB frames have been the subject of research for many years (e.g. Humason et al. 1956; Yahil et al. 1977, Sandage 1986, Karachentsev \& Makarov 1996; Rauzy \& Gurzadyan 1998: Courteau \& van den Bergh 1999 van den Bergh 2000). Obvious galaxy members of the LG are M31 and the MW. The assignment of other members to the LG is based on the assumption that they form a bound object detached from the general cosmic expansion. Therefore, the LG motion is found by fitting a constant velocity to the radial velocities (redshifts) of LG galaxies measured with respect to the Local Standard of Rest (defined by mean motion of stars in the solar neighborhood). The criteria of whether or not a galaxy belongs to the LG depends on the LG velocity itself. Hence the procedure is essentially iterative. An LG member galaxy must satisfy the following criteria (e.g. Yahil et al. 1977; Lynden-Bell 1981; Courteau \& van den Bergh 1999):

- it should not appear to be associated with any other group of galaxies;

- its distance from the LG barycenter should be smaller than the radius of the surface of zero velocity (with respect to the LG velocity).

- its radial velocity does not deviate significantly from the value obtained from the constant velocity fit.

A recent analysis (Mikulizky \& Nusser 2013) yields 14 well-established members within $\sim 1.4 \mathrm{Mpc}$ from the LG barycenter, not including satellites of M31 and MW. The velocity of LG in the CMB frame from this analysis is 
$622 \pm 33 \mathrm{kms}^{-1}$ in the $l=277^{\circ} \pm 3^{\circ}$ and $b=33^{\circ} \pm 3^{\circ}$ direction, in agreement with Kogut et al. (1993). In the standard paradigm for structure formation, this motion should be the result of the cumulative gravitational tug of matter in the universe. The subject of the paper is to assess how well the gravitational force field matches the LG velocity measured in the CMB.

\section{LINEAR THEORY OF THE LG MOTION}

We assume that all quantities are given at the present time with the expansion scale factor $a$ set to unity so that the comoving and physical distances are equal. Let $\boldsymbol{r}$ be the real space coordinate (proper distance) and $\boldsymbol{v}=\mathrm{d} \boldsymbol{r} / \mathrm{d} t$ the corresponding peculiar velocity of a patch of matter. We shall assume that the mass density contrast, $\delta_{d m}$, is related to the galaxy (number) density contrast, $\delta$, by a linear biasing relation $\delta=b \delta_{d m}$. The linear theory for structure formation (e.g. Peebles 1980) relates the divergence of the peculiar velocity field, $\boldsymbol{v}(\boldsymbol{r})$, to the density contrast as

$$
\boldsymbol{\nabla} \cdot \boldsymbol{v}=-H_{0} \beta \delta,
$$

where $H_{0}$ is the Hubble constant, $\beta=f / b$ and $f=$ $\operatorname{d} \ln D / \operatorname{dn} a$ is the logarithmic derivative of the linear growth rate with respect to the scale factor, $a$.

Observations provide the angular positions and redshifts of galaxies, $c z=H_{0} r+\hat{\boldsymbol{r}} \cdot \boldsymbol{v}$ where $\hat{\boldsymbol{r}} \cdot \boldsymbol{v}$ is the radial peculiar velocity, and $\hat{\boldsymbol{r}}$ denotes the direction of vector $r$. Hence, for the realistic reconstruction of velocities from redshift space data, the relation (1) needs to be modified in order to account for the added displacement from $r$ to $c z$. We define the redshift space coordinate,

$$
\boldsymbol{s} \equiv \boldsymbol{r}+H_{0}^{-1}(\hat{\boldsymbol{s}} \cdot \boldsymbol{v}) \hat{\boldsymbol{s}}
$$

where $\hat{\boldsymbol{s}}=\hat{\boldsymbol{r}}$.

Let $n^{s}(\boldsymbol{s})=\bar{n}\left[1+\delta^{\mathrm{s}}(\boldsymbol{s})\right]$ and $n(\boldsymbol{r})=\bar{n}[1+\delta(\boldsymbol{r})]$ be, respectively, estimates of the number densities of galaxies in redshift and real space, where $\bar{n}$ is the mean number density of galaxies in the survey (assumed to be the same in both spaces). In the limit $\delta \ll 1$, the mapping (2) and the continuity equation $n^{s}(\boldsymbol{s}) \mathrm{d}^{3} s=n(\boldsymbol{r}) \mathrm{d}^{3} r$ modifies the real space linear equation (1) as

$$
\boldsymbol{\nabla} \cdot \boldsymbol{v}+\beta \boldsymbol{\nabla} \cdot[(\hat{\boldsymbol{s}} \cdot \boldsymbol{v}) \hat{\boldsymbol{s}}]=-H_{0} \beta \delta^{\mathrm{s}} .
$$

To linear order, $\delta^{\mathrm{s}}(\boldsymbol{s})=\delta^{\mathrm{s}}(\boldsymbol{r})$ and similarly for $\delta$ and $\boldsymbol{v}$. Given appropriate boundary conditions, a unique solution to this equation can be obtained for a potential flow, $\boldsymbol{v}=-\boldsymbol{\nabla} \phi$, where $\phi$ is a scalar function of the spatial coordinates. For our purposes, it is convenient to express the solution in terms of the spherical harmonics, $Y_{l m}$, expansion of the angular dependence of $\phi(\boldsymbol{r})$ and $\delta(\boldsymbol{r})$. Writing

$$
\phi(\boldsymbol{r}) \equiv \phi(r, \hat{\boldsymbol{r}})=\sum_{l \geq 0} \sum_{\mathrm{m}=-l}^{l} \phi_{l m}(r) Y_{l m}^{*}(\hat{\boldsymbol{r}})
$$

where $\hat{\boldsymbol{r}}$ is the radial unit vector, $r=|\boldsymbol{r}|$ the distance. $\delta(r, \hat{\boldsymbol{r}})$ is similarly expanded. The solution to (3) is
(Nusser \& Davis 1994)

$$
\begin{aligned}
\phi_{l m}(s) & =-\frac{H_{0} \beta /(1+\beta)}{2 \tilde{l}+1}\left[s^{\tilde{l}} \int_{s}^{s_{1}} \frac{\delta_{l m}^{\mathrm{s}}(u)}{u^{\tilde{l}-1}} \mathrm{~d} u\right. \\
& \left.+\frac{1}{s^{\tilde{l}+1}} \int_{0}^{s} \delta_{l m}^{\mathrm{s}}(u) u^{\tilde{l}+2} \mathrm{~d} u\right],
\end{aligned}
$$

where $\tilde{l} \leq l$ is related to the harmonic order $l$ through the algebraic equation $(1+\beta) \tilde{l}(\tilde{l}+1)-l(l+1)=0$, and $s_{1}$ is a constant dictated by the appropriate boundary conditions. The solution in real space is obtained in the limit $\beta \ll 1$ where $\tilde{l} \rightarrow l$ and $\beta /(1+\beta) \rightarrow \beta$. Only the dipole, $l=1$, component is relevant for the LG motion. For this component, the appropriate choice is $s_{1}=0$. Thus we work in the freely falling LG frame, not the $\mathrm{CMB}$ frame, because obtaining the CMB should be a result of the analysis. After all, we don't know how large is the sphere around us that has the same dipole CMB anisotropy. Further, working with CMB redshifts causes a singular behavior of the density at the origin. In the LG frame, the mean motion of a very distant thin spherical shell, i.e. the reflex dipole, approaches the negative of the LG motion in the CMB frame. Thus the LG motion in the CMB frame, $\boldsymbol{v}_{\mathrm{lg}}$, is identified with the negative of the reflex dipole of a very distant shells. In $\mathrm{A}$ we derive a new relation for relating $\boldsymbol{v}_{\mathrm{lg}}$ to the density distribution in redshift space.

For a sampling of the density in redshift space by a discrete distribution of $N$ mass tracers (galaxies) with redshift coordinates $\boldsymbol{s}_{i}(i=1 \cdots N)$ the relation yields

$$
\begin{aligned}
\boldsymbol{v}_{\mathrm{lg}}\left(R_{\mathrm{out}}\right)= & \frac{R_{\mathrm{out}}^{\tilde{l}-1}}{(1+\beta)} \frac{\beta H_{0}}{4 \pi \bar{n}} \sum_{R_{\mathrm{lg}}<s_{i}<R_{\mathrm{out}}} \frac{\boldsymbol{s}_{i}}{\varphi_{i} s_{i}^{\tilde{l}+2}} \\
& -\frac{(1-\tilde{l}) R_{\mathrm{out}}^{-(\tilde{l}+2)}}{(1+\beta)(2 \tilde{l}+1)} \frac{\beta H_{0}}{4 \pi \bar{n}} \sum_{R_{\mathrm{lg}}<s_{i}<R_{\mathrm{out}}} \frac{s_{i}^{\tilde{l}-1}}{\varphi_{i}} \boldsymbol{s}_{i},
\end{aligned}
$$

where $\tilde{l}$ correspond to $l=1$, i.e. it is the solution to $(1+\beta) \tilde{l}(\tilde{l}+1)-2=0$, the selection function $\varphi_{i}$ compensates for the missing faint galaxies in flux limited surveys and $\bar{n}$ is a measure of the average number density of galaxies. The sum extends over all tracers between $R_{\mathrm{lg}}$, the radius assigned to the $\mathrm{LG}$, and a maximum distance $R_{\text {out }}$. In principle all fluctuations out to $R_{\text {out }} \rightarrow \infty$ contribute to $\boldsymbol{v}_{\mathrm{lg}}$. In a hierarchical model for structure formation, distant structures typically make smaller contributions. It is therefore, interesting to study the convergence of $\boldsymbol{v}_{\mathrm{lg}}$ as a function of $R_{\text {out }}$. Further, in realistic redshift surveys, the noise increase dramatically at large redshifts, due the significant decrease in the number of observed galaxies and the recovery of $\boldsymbol{v}_{\mathrm{lg}}$ can be assessed reliably only out to the radius beyond which the large scale structure is poorly probed.

The real space counterpart of the relation $(6)$ is obtained by setting $\tilde{l}=l=1$ and replacing $f$ with $\beta /(1+\beta)$. This yields

$$
\boldsymbol{v}_{\mathrm{lg}}=\frac{H_{0} \beta}{4 \pi \bar{n}} \sum_{R_{\mathrm{out}}>r_{i}>R_{\mathrm{lg}}} \frac{\boldsymbol{r}_{i}}{\varphi_{i} r_{i}^{3}},
$$

where we use the same symbol $R_{\text {out }}$ to indicate the maximum distance in real space. Note the disappearance of 
the counterpart of the second term on the r.h.s of equation (6). However, even for redshift space reconstruction by equation (6), the second term makes negligible contribution to $\boldsymbol{v}_{\lg }$ as we find in the numerical tests below.

\section{CONSTRUCTION OF MOCK GALAXY CATALOGS}

We consider mock catalogs designed to match the 2MRS catalog of $\sim 45000$ galaxies with $K_{s} \leq 11.75$ (Huchra et al. 2012). A parent simulated catalog of the whole 2MASS catalog has been prepared (De Lucia \& Blaizot 2007) by incorporating semi-analytic galaxy formation models in the Millennium simulation (Springel et al. 2005) of the $\Lambda$ CDM model with $\Omega=0.25, \Omega_{b}=$ $0.045, \sigma_{8}=0.9, \Lambda=0.75$, and $H_{0}=73 \mathrm{~km} \mathrm{~s}^{-1} \mathrm{Mpc}^{-1}$. From this parent catalog we have drawn 53 independent mock 2MRS catalogues satisfying the following conditions:

- The "observer" in each mock is selected to reside in a galaxy with a quiet velocity field within $500 \mathrm{~km} \mathrm{~s}^{-1}$, similar to the observed universe. One observational signature of this quiet flow is that in the LG frame, the only galaxies with measured negative redshift belong to the Virgo cluster. We enforce this condition by imposing that the observer sees at most one cluster that has high enough peculiar velocities to result in negative redshifts.

- Note that in finding a cluster which produces negative redshifts in its core region necessarily implies the average overdensity toward the region is substantial. For example, the average overdensity towards the Virgo cluster is $\delta \sim 2$ (Davis \& Huchra 1982), and the mock catalogs are roughly the same. That means that the flows in this direction are becoming nonlinear. To keep the overdensity close to that of the Virgo cluster, we select only clusters with mean overdensity between the LG and the cluster center to be $\delta<2$. This is quite a stringent constraint that, alone, eliminates $\sim 70 \%$ of the potential LG "observers".

- The velocity of the central galaxy must be in the range 400 to $700 \mathrm{~km} \mathrm{~s}^{-1}$ to match that of the LG with respect to the $\mathrm{CMB}$.

- The density in the LG environment, i.e. averaged over a sphere of $5 \mathrm{Mpc}$ radius around the observer, is less than twice the cosmic mean.

- We impose the constraint that the bulk flow of a sphere of radius, $3.5 h^{-1} \mathrm{Mpc} \approx 5 \mathrm{Mpc}(h=$ $\left.H_{0} /\left[100 \mathrm{~km} \mathrm{~s}^{-1} \mathrm{Mpc}\right] \approx 0.7\right)$, centered on the LG, is also in the range $400-700 \mathrm{~km} \mathrm{~s}^{-1}$. This radius is more than three times larger than the radius assigned to the real $\mathrm{LG}(\sim 1.4 \mathrm{Mpc})$. This additional constraint is meant to eliminate strong nonlinearities that may still be present in some of the mocks after applying the previous constraints mentioned above. This choice is justified by the fact that strong nonlinearities inside $5 \mathrm{Mpc}$ seem to be absent in the real Universe, as indicated by the fact that the flow is fairly quiet within that radius. Hereafter we take $R_{\mathrm{lg}}=5 \mathrm{Mpc}$ as the radius of the LG and treat the motion of of the central sphere of that radius as the motion of the LG.

The mocks are taken from the $z=0$ simulation output and, therefore, are free from any possible galaxy evolution. The 2-point correlation function of the mock galaxies fits reasonably well the observed one (Westover 2007), but less so is the K-band luminosity function, resulting in a discrepancy with the observed number of galaxies. To fix the problem, the original luminosity of mock galaxies was shifted to brighter values by $\sim 1.5$ magnitude. We obtained, on average, $\sim 50000$ galaxies per mock, slightly larger than but close to the real $K_{s}=11.752 \mathrm{MRS}$ catalog. Each of the 53 mock catalogs contains galaxy distances, peculiar velocities (and hence redshifts), angular positions and $K_{s}$-band magnitudes.

\subsection{The selection function}

In the application to a flux limited survey like $2 \mathrm{MRS}$, each galaxy in the summation in the relations (6) and (6) should be weighted by the inverse of the selection function, $\varphi$, to compensate for missing faint galaxies that fall below the flux limit. The selection function depends on the galaxy distances and it is physically determined by the distribution of galaxy luminosities. In the mocks, where galaxy distances and apparent magnitude are both known, we compute $\varphi$ using a direct method which avoids the explicit calculation of the luminosity function (Turner 1979, Kirshner et al. 1979, Davis $\&$ Huchra 1982). The method provides discrete values of $\varphi$ in distance bins, which are then interpolated to the galaxy distances to yield the weights to be assigned to individual galaxies. In realistic applications, however, the distances to the galaxies are not known. Using redshifts rather than distances as arguments to the selection function induces systematic errors, sometimes dubbed as "Kaiser Rocket" effect (Kaiser 1987). The hazards of not accounting explicitly for the Kaiser rocket effect are given in 86 .

\subsection{Bias of the selected galaxies}

Galaxies typically form at the peaks of the mass density field and, therefore, are not unbiased tracers of the underlying density field. An indirect but strong evidence for galaxy biasing is the fact that different types of object exhibit different clustering properties. On large scales, however, it is safe to assume a linear biasing relation between the density contrast of the matter and the galaxy distribution, $\delta$ (galaxies) $=b \delta$ (mass), with a constant bias factor $b$. If biasing is a local, though not necessarily a Poisson, process, then this form is motivated by theory on linear scales (e.g. Kaiser 1988; Coles 1993; Fry \& Gaztanaga 1993, Scherrer \& Weinberg 1998; Coles et al. 1999, Seljak 2001, Smith et al. 2007), confirmed by numerical experiments (e.g. Kauffmann et al. 1997; Narayanan et al. 2000, Benson et al. 2000, Huff et al. 2007) and supported by observations involving galaxy samples dominated, like in the $2 \mathrm{MRS}$ case, by late type galaxies (e.g. Tegmark et al. 2001; Verde et al. 2002, Westover 2007).

We explore here the bias of the distribution of the mock galaxies with respect to the dark matter density field in the simulation. Gerard Lemson has kindly used the facilities of the Millennium Simulation Database to 


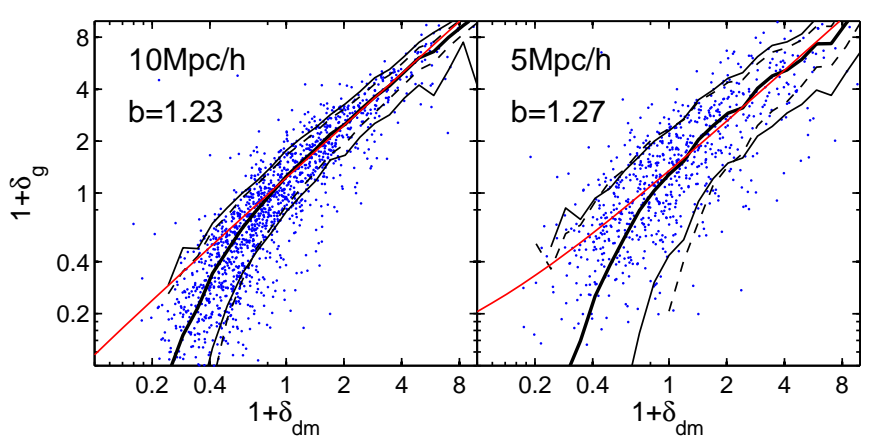

FIG. 1.- A scatter plot (logarithmic scale) of the galaxy versus the dark matter over-densities in the simulation. For each of the 53 mocks, densities of 125 randomly selected points with a distance $<70 h^{-1} \mathrm{Mpc}$ from the LG candidate, are shown. The left and right panels correspond to densities in cubic cells of $10 h^{-1} \mathrm{Mpc}$ and $5 h^{-1} \mathrm{Mpc}$ on the side, respectively. The thick solid curve in each panel is the mean of $1+\delta_{\mathrm{g}}$ at a given $1+\delta_{\mathrm{dm}}$. The two thin solid curves are $\pm 1 \sigma$ scatter computed from points above and below the mean. Dashed curves are the expected $\pm 1 \sigma$ Poisson (shot-noise) scatter. The nearly straight red lines show $\delta_{\mathrm{g}}=b \delta_{\mathrm{dm}}+$ const, where $b$ (indicated in the figure) are determined using linear regression from points in the range $-0.5<\delta_{\mathrm{dm}}<4$.

produce for us the density field from all $2160^{3}$ dark matter particles in the simulation box on a cubic grid of $1 h^{-1} \mathrm{Mpc}$ spacing. Density fields from the distribution of mock galaxies have also been computed directly for all the mocks. Figure (1) is a scatter plot of the over densities computed from the mock galaxy distribution versus the dark meter density field. For $\delta_{\mathrm{dm}} \lesssim 3$, the scatter in the relation is mainly Poissonian. However, at higher densities, intrinsic scatter in the biasing relation dominates. The relation in small (right panel) and large (left) cells is fairly linear, $\delta_{\mathrm{g}}=b \delta_{d m}$, in the moderate density $\left(-0.2 \lesssim \delta_{\mathrm{dm}} \lesssim 4\right.$ in the two panels) regions, with a weak dependence of $b$ scale: $b \sim 1.23$ and 1.27 in the large and small cells, respectively. The values change according to the density cut used in fits. Exploration of $b$ for various densities yields $1.2<b<1.35$ as an acceptable range. We shall continue to assume linear bias, bearing in mind that it breaks down in deep voids.

\section{RECONSTRUCTION OF THE LG MOTION}

In this section we tests the ability to reconstruct the LG velocity using the relations $(6 \sqrt[6]{6})$. We first consider the ideal case in which we can perform the reconstruction using the dark matter density field. The aim of this test is to assess the possibility of reconstructing $\boldsymbol{v}_{\mathrm{lg}}$ and to estimate the impact of shot noise errors. In the second part we repeat the reconstruction procedure on the realistic 2MRS mocks described in Section 4. The goal here is twofold: to assess our ability to determine $\beta$ by comparing the predicted LG motion with the true LG velocity and to measure the accuracy with which one can predict $\boldsymbol{v}_{\mathrm{lg}}$ when the $\beta$ is given a priori.

\subsection{Reconstruction tests using the full dark matter distribution}

Given the location of each mock LG in the parent simulation we use the actual density field $\delta_{\mathrm{dm}}$ to recover the motion of the corresponding LG. We perform this test only in real space, by adapting equation (6) to density fields given on a grid, i.e.

$$
\boldsymbol{v}_{\mathrm{lg}}\left(R_{\text {out }}\right)=\frac{H_{0} \beta}{4 \pi} \sum_{R_{\text {out }}>R_{\alpha}>R_{\mathrm{lg}}} \delta_{\alpha} \frac{\boldsymbol{r}_{\alpha}}{r_{\alpha}^{3}}
$$

where the summation is over the grid points, $\boldsymbol{r}_{\alpha}$ is the distance of the grid cell $\alpha$ from the LG position and $\delta_{\alpha}$ is $\delta_{d m}$ in the cell $\alpha$. We apply the relation (7) with the largest possible outer radius, namely $R_{\text {out }}=250 h^{-1} \mathrm{Mpc}$. Further, to minimize the influence of mass fluctuations beyond $R_{\text {out }}$ we measure $\boldsymbol{v}_{\text {lg }}$ with respect to the bulk flow of the sphere. Because of the missing power on scales $>$ $500 h^{-1} \mathrm{Mpc}$ in the simulation, this is only $10-30 \mathrm{~km} \mathrm{~s}^{-1}$ and hence this last step has little effect on our results.

Since we are dealing with the dark matter directly and are assuming a flat $\Lambda$ CDM model, we have $\beta=f \approx$ $\Omega_{m}^{0.55}=0.466\left(\right.$ Linder 2005) for $\Omega_{m}=0.25$ of the simulation. However, due to nonlinear effects, we do not expect the reconstructed $\boldsymbol{v}_{\mathrm{lg}}$ to coincide exactly with its true value. We obtain an estimate of $\beta$ in each mock by matching the motion recovered with $\beta=f, \boldsymbol{v}_{\mathrm{lg}}^{\text {rec }}$, with the true motion, $\boldsymbol{v}_{\mathrm{lg}}^{\text {tru }}$ :

$$
\frac{\beta}{f}=\frac{\left|\boldsymbol{v}_{\mathrm{lg}}^{\mathrm{tru}}\right|}{\hat{\boldsymbol{v}} \cdot \boldsymbol{v}_{\mathrm{lg}}^{\mathrm{rec}}},
$$

Here $\hat{\boldsymbol{v}}$ is a unit vector in the direction of the true motion and $\hat{\boldsymbol{v}} \cdot \boldsymbol{v}_{\mathrm{lg}}^{\mathrm{rec}}=v_{\mathrm{lg}, \|}^{\mathrm{rec}}$ is the parallel component of $\boldsymbol{v}_{\mathrm{lg}}^{\mathrm{rec}}$ in the direction of $\boldsymbol{v}_{\mathrm{lg}}^{\mathrm{tru}}$. If the reconstruction error, $\sigma$, scales like $\beta$, as it should, then this estimate of $\beta$ minimizes the quantity

$$
\begin{aligned}
\chi^{2}(\beta) & =\sigma^{-2}\left[\boldsymbol{v}_{\mathrm{lg}}^{\mathrm{tru}}-\frac{\beta \boldsymbol{v}_{\mathrm{lg}}^{\mathrm{rec}}}{f}\right]^{2} \\
& =\sigma^{-2}\left[\left(\frac{\beta \boldsymbol{v}_{\mathrm{lg}, \perp}^{\mathrm{rec}}}{f}\right)^{2}+\left(v_{\mathrm{lg}}^{\mathrm{tru}}-\frac{\beta v_{\mathrm{lg}, \|}^{\mathrm{rec}}}{f}\right)^{2}\right] .
\end{aligned}
$$

The solid, black histogram in Figure (2) shows the distribution of $\beta / f$ values obtained using Equation (8). The mean value of $\beta / f$ from the 53 mocks is 0.93 and the $1 \sigma$ scatter is 0.085 . This is a remarkable result considering that the value of $\beta$ results from a comparison at a single point, i.e. the gravity acceleration at the position of the LG candidate versus velocity of the LG. The slight downward bias of $\beta$ with respect to the expected value, $f$, is due to minor non-linear dynamical effects which persist even in the quiet environment of the LG candidates. Since linear theory typically yields larger predicted velocity amplitude (Nusser et al. 1991), a smaller value of $\beta$ is obtained from the comparison of the linear prediction with the true velocities. This deviation from linear theory is less than $10 \%$ and would be difficult to detect in any actual test of the LG.

The black curves in figure (3) are the residuals between true and recovered $\boldsymbol{v}_{\mathrm{lg}}$ for 9 randomly selected mocks, using the full dark matter density. In the reconstruction we have adopted $\beta=\beta_{\mathrm{dm}}=0.927 f=0.43$ for all the mocks. The solid curve corresponds to the parallel component, $v_{\mathrm{lg}, \|}^{\mathrm{tru}}-v_{\mathrm{lg}, \|}^{\mathrm{rec}}$ of the residuals and the dashed is the amplitude of the perpendicular residual, $\boldsymbol{v}_{\mathrm{lg}, \perp}^{\mathrm{tru}}$. Both the parallel and perpendicular residuals change rapidly for $R_{\text {out }} \lesssim 100 h^{-1} \mathrm{Mpc}$ and both flatten 


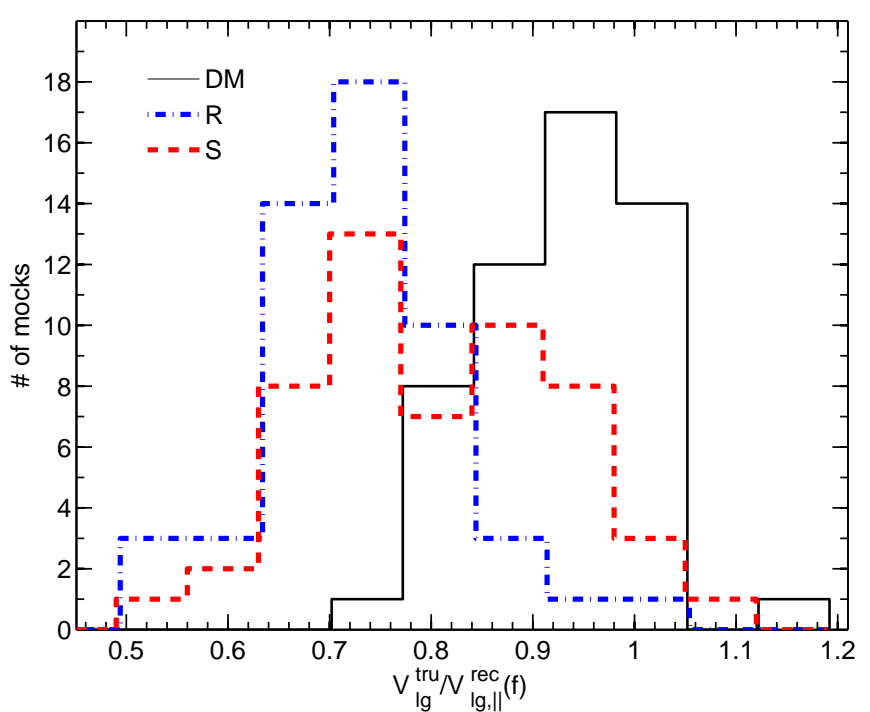

FIG. 2.- Histograms of the distribution of the ratio $v_{\mathrm{lg}}^{\text {tru }}$ to the $v_{\mathrm{lg}}^{\text {rec }}$ recovered with a value of $\beta=f$, i.e. no bias. Black solid, blue dot-dashed and red dashed curves correspond, respectively, to reconstructions from the full dark matter density in real space, $2 \mathrm{MRS}$ mocks in real space, and in redshift space. For the real space reconstructions, this ratio equals $\beta / f$ (see eq. 8). For redshift space, the ratio depends non-linearly on $\beta / f$ as explained in $\delta$. The averages and standard deviations are $(0.72,0.12),(0.80,0.09)$ and $(0.93,0.08)$, respectively, for the red, blue, and black histograms. Deviations of the mean values from unity quantify the systematic errors and random uncertainties in the reconstructions. Note that once we account for galaxy bias in the mocks, the mean $\beta$ values obtained from the histograms are consistent with each other.

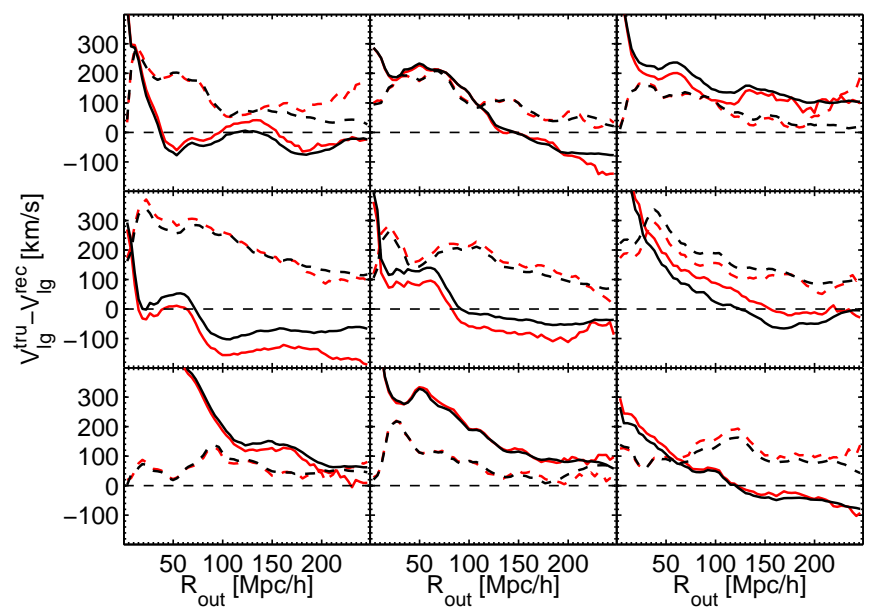

FIG. 3.- LG motions recovered in full density distribution versus dilute distribution of galaxies as a function of $R_{\text {out }}$. As $R_{\text {out }}$ increases, the velocity residuals should approach zero; they do shrink but often asymptote at a value $\sim 100 \mathrm{~km} / \mathrm{s}$. The parallel (to the true motion) and perpendicular components of the residuals are shown as the solid and and dashed lines, respectively. Black curves correspond to reconstruction from the full dark matter density field, while the red curves are obtained by resampling the dark matter with a distance dependent number density of tracers as appropriate for the $2 \mathrm{MRS}$.

at $R_{\text {out }} \gtrsim 150-200 h^{-1} \mathrm{Mpc}$. This shows the danger of working in the $\mathrm{CMB}$ frame, as deviations from it are substantial up to $R_{\text {out }} \sim 50 h^{-1} \mathrm{Mpc}$.

The residual at $250 h^{-1} \mathrm{Mpc}$ is entirely due to dynamical errors in the reconstruction. The $\mathrm{rms}$ values of the parallel and perpendicular residuals, at $R_{\text {out }}=$ $250 h^{-1} \mathrm{Mpc}$, from all 53 mocks are $54 \mathrm{kms}^{-1}$ and
$76 \mathrm{kms}^{-1}$, respectively. The rms of the total residual, $\boldsymbol{v}_{\mathrm{lg}}^{\mathrm{tru}}-\boldsymbol{v}_{\mathrm{lg}}^{\mathrm{rec}}$, is $\sigma_{\mathrm{dyn}}=94 \mathrm{~km} \mathrm{~s}^{-1}$.

\subsubsection{The impact of shot-noise}

In order to assess the Poissonian (shot-noise) error introduced by the finite number of galaxies in the mocks, we take each of the 53 mocks and diluted the dark matter distribution around its LG candidate with a radial distribution that follows the selection function of the corresponding $2 \mathrm{MRS}$ mock. For each of these dark matter particles-only mocks, we apply the real space relation (6) with $\beta=\beta_{\mathrm{dm}}=0.43$ to derive $\boldsymbol{v}_{\mathrm{lg}}$. The red curves in figure (3) are the residuals between true and recovered $\boldsymbol{v}_{\mathrm{lg}}$ obtained from the dilute dark matter particles for the same mocks as the black curves. The solid and dashed curves indicate residuals in the parallel and perpendicular directions, respectively. The red and the corresponding black curves agree very well, indicating that the shot noise contribute to random uncertainties but does not not introduce systematic errors, as expected. The $\mathrm{rms}$ of the difference between the reconstructed $\boldsymbol{v}_{\mathrm{lg}}$ with and without shot-noise is $\sigma_{\mathrm{sn}}=90 \mathrm{~km} \mathrm{~s}^{-1}$. This is comparable to $\sigma_{\text {dyn }}=94 \mathrm{~km} \mathrm{~s}^{-1}$ of dynamical inaccuracies in the reconstruction as discussed in the previous subsection. Note that the shot-noise error scales linearly with $\beta$ for real space reconstruction.

Another way to estimate the shot-noise error is by bootstrap resampling of the galaxy distribution in the mocks. This yields the following estimate for this error in each mock,

$$
\sigma_{s n} \approx \frac{H_{0} f}{4 \pi \bar{n}}\left[\sum_{R_{\mathrm{out}}>r_{i}>R_{\mathrm{lg}}} \frac{1}{\varphi_{i}^{2} r_{i}^{4}}\right]^{1 / 2} .
$$

Both estimates yield similar values.

The number density of $2 \mathrm{MRS}$ galaxies is representative of that in existing and planned spectroscopic galaxy redshift surveys. A significant reduction of shot noise, say a factor of 2 , would require increasing the number density of objects by a factor of 4 which, using the luminosity function in Branchini et al. (2012), means pushing the magnitude limit of the redshift survey about one magnitude fainter.

\subsection{Reconstruction tests using the realistic 2MRS mock catalogs}

We now turn to the reconstruction of LGs from the distribution of synthetic galaxies in the 53 2MRS mocks. We perform the reconstruction both in redshift and real space, from equations (6) and (6), respectively. Galaxies are assigned weights according to the selection function as outlined in 4.1 . As before, we remove the effect of external fluctuations beyond the sphere of radius $250 h^{-1} \mathrm{Mpc}$ around each mock by measuring the LG motion relative to the bulk flow of the sphere. Since we want to focus on the ability of linear theory to recover the LG velocity we shall initially ignore the Kaiser rocket effect and consider the selection function estimated in real space, deferring the additional complication related to the estimation of the selection function in redshift space to 6 . 
TABLE 1

Mean Values and STANDARD DEVIations of the Distributions FOR $v_{\mathrm{lg}}^{\mathrm{tru}} / v_{\mathrm{lg}, \|}^{\mathrm{rec}}\left(\beta_{0}=f\right)$ COMPUTED FROM THE 53 MOCKS AND Shown in Figure 2 Col.1: Tracers. Col 2: Type of ReCONSTRUCTION (REAL OR REDShift SPACE). COL 3: MEAN value. Col 4: Standard deviation.

\begin{tabular}{|c|c|c|c|}
\hline \hline Tracer & Space & Average & Variance \\
\hline Dark Matter & Real & 0.93 & 0.08 \\
Mock Galaxies & Real & 0.80 & 0.09 \\
Mock Galaxies & Redshift & 0.72 & 0.12 \\
\hline
\end{tabular}

\subsubsection{Estimation of $\beta$ by matching the recovered and true $L G$ motions}

We pursue the same strategy as in $\$ 5.1$ and determine the values of $\beta$ by requiring zero residuals in the parallel components of the reconstructed LG velocity for each of the 53 mocks. In this way we gauge how accurately $\beta$ can be assessed by matching the gravity field to observed LG motion.

We reconstruct the LG motion in real and redshift space using Equations (6) and (6), respectively, summing over all mock galaxies within $R_{\text {out }}=250 h^{-1} \mathrm{Mpc}$ and using $\beta_{0}=f$ as a reference value. This corresponds to assuming that linear theory applies and that galaxies are unbiased tracers of the mass. The results of this test are shown in Figure 2 in the form of histograms. They represent the distribution of the ratio $v_{\mathrm{lg}}^{\mathrm{tru}} / v_{\mathrm{lg}, \|}^{\mathrm{rec}}\left(\beta_{0}=f\right)$ measured in the 53 mock catalogs. These histograms are analogous to that obtained in Section 5.1 (black solid histogram) but refer to mock galaxies in real (blue, dotdashed curve) and and redshift (red dashed) space rather than to dark matter particles.

In real space these histograms represent the distribution of $\beta / f$, according to (8). In redshift space the link between the recosntructed LG mostion and the value of $\beta$ is less straightforward since, in this case, peculiar velocities do not scale linearly with $\beta$, as we show explicitly in Appendix B. The mean values and the scatter of each histogram are listed in Table 1

The three histograms in the plot are not expected to match for several reasons. First of all, as previously discussed, linear theory is not quite able to describe the LG motion. Not even using the full dark matter particles population in real space. This explains why the peak of the solid curve is at $\sim 0.9$, rather than 1 . Secondly, galaxy bias induces a systematic mismatch between the value $\beta_{\mathrm{dm}}$ obtained from dark matter particles and the one obtained from mock galaxies in real space, $\beta_{\mathrm{r}}$ that should be equal to the linear bias parameter of the sample $\beta_{\mathrm{r}} / \beta_{\mathrm{dm}} \equiv b$. Indeed, we find that the value of this ratio $(=1.28)$ is consistent with the value of the linear bias in the mocks $(1.2-1.35)$ obtained from the scatterplot (1) in 8.2 . This is a remarkable results since it shows that comparing gravity and velocity in a single LG-like region can provide an unbiased, if noisy, estimate of $\beta$.

Additional errors caused by performing the reconstruction in redshift rather than real space are the origin of the mismatch between the red-dashed and the blue, dotdashed histograms. Remarkably, the differences between the two curves are not large. This is very welcome as putting all the galaxies into real space is problematic; it is easier to leave the galaxies in redshift space.

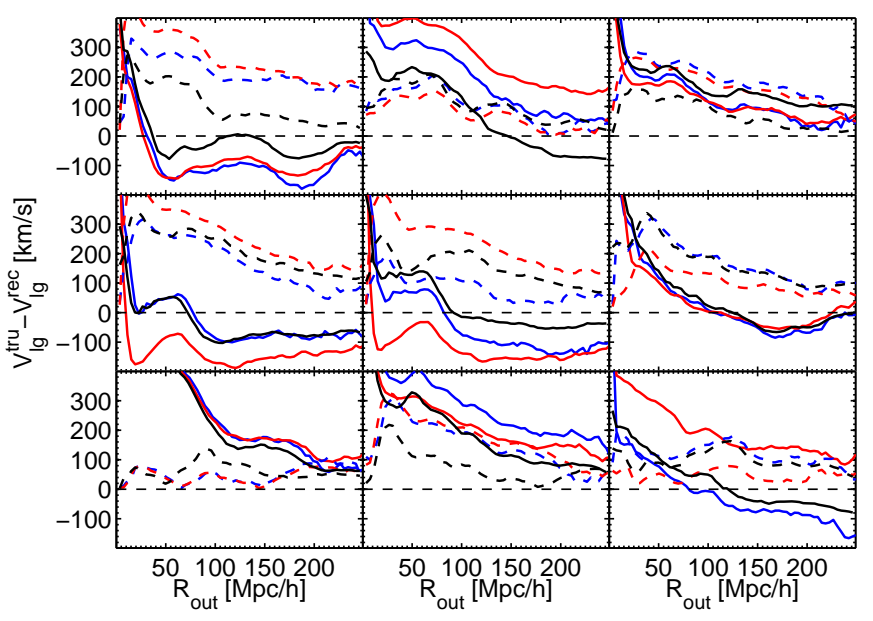

FIG. 4. - Residuals of LG motions for the same 9 mocks in figure 3 for reconstructions from the galaxy distribution in real (blue curves) and redshift (red) space. For reference we also plot the black curves of figure (3) that represent the dark matter case. Parallel and perpendicular components of the residuals are shown as the solid and and dashed lines, respectively.

Assuming that in the observations $\beta$ is determined as outlined above, we further ask how well the direction of the LG motion can be recovered. The individual $\beta$ values above yield $v_{\mathrm{lg}, \|}^{\mathrm{rec}}=v_{\mathrm{lg}, \|}^{\mathrm{tru}}$ and, therefore, the angle, $\theta$, between $\boldsymbol{v}_{\mathrm{lg}}^{\text {rec }}$ and $\boldsymbol{v}_{\mathrm{lg}}^{\text {tru }}$ is

$$
\theta=\tan ^{-1} \frac{v_{\mathrm{lg}, \perp}^{\mathrm{rec}}}{v_{\mathrm{lg}, \|}^{\mathrm{tru}}} .
$$

This yields $<\theta^{2}>^{1 / 2} \approx 10^{\circ}$ for real as well as redshift space reconstruction.

$$
\text { 5.2.2. } \boldsymbol{v}_{\mathrm{lg}}^{\mathrm{rec}} \text { versus } \boldsymbol{v}_{\mathrm{lg}}^{\mathrm{tru}} \text { for a fixed } \beta
$$

We now assess the goodness of the LG velocity reconstruction, in both real and redshift space, when the value of $\beta$ is given a priori. We do this in three steps. First we set a convenient value of $\beta$. Then we reconstruct $\boldsymbol{v}_{\mathrm{lg}}$ using all objects within $R_{\text {out }}=250 h^{-1} \mathrm{Mpc}$ in each of the 53 mocks. And finally we compare the result with the true LG motion. We set $\beta$ in correspondence of the mean values of the histograms shown in Figure 2, i.e. $\beta_{\mathrm{r}}=0.33$ and $\beta_{\mathrm{s}}=0.25$ in real and redshift space, respectively. This guarantees that the reconstructed parallel LG velocities are evenly distributed around the true values.

The results are shown in Figure (4), which is the analogous of Figure (3). The 9 panels refer to the same mocks of that plot. Blue and red curves refer to reconstructions performed in real and redshift space, respectively. The black curves are the same of Figure (3) and show the case of dark matter reconstruction. Moreover the residuals in redshift space are occasionally much smaller than in real space. This is an additional confirmation that there is no problem in performing the computation in redshift space (Nusser \& Davis 1994). Finally, we note that beyond $150 h^{-1} \mathrm{Mpc}$ the curves become flat, indicating that most of the contribution to $\boldsymbol{v}_{\mathrm{lg}}^{\mathrm{rec}}$ arises from large scale structure within that radius.

Figure (5) provides an additional assessment of the goodness of the reconstruction. It shows the parallel 


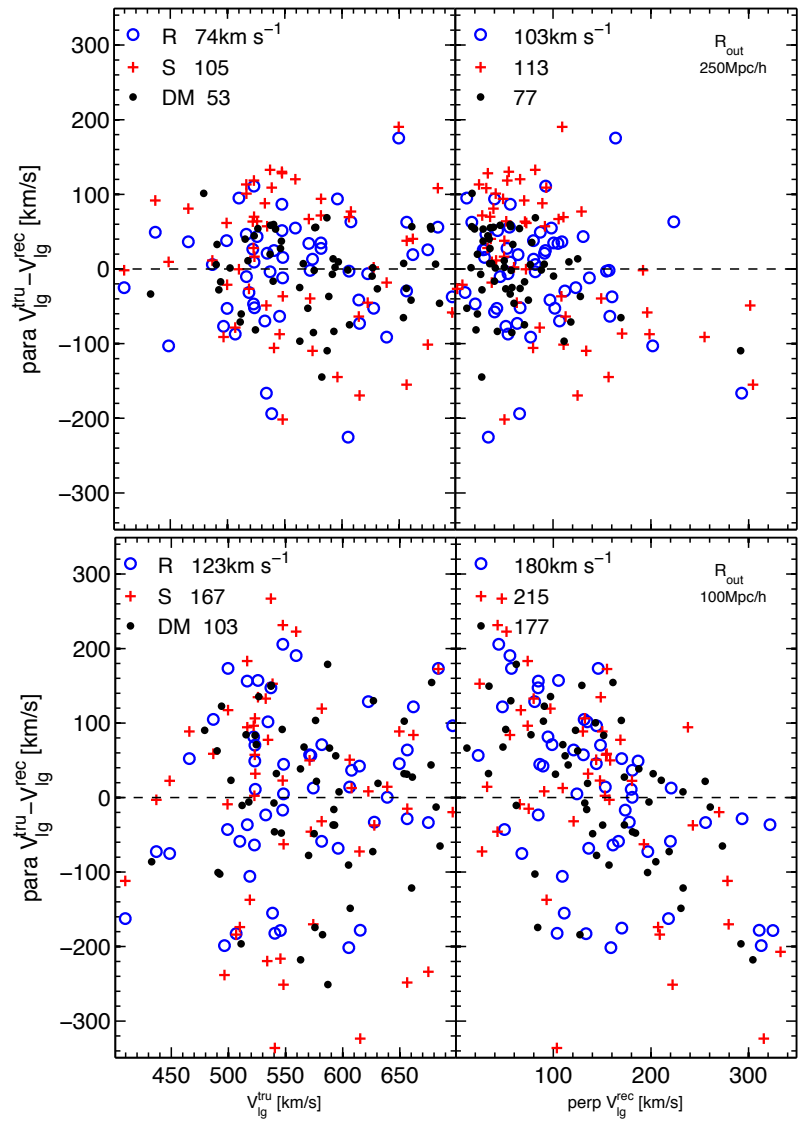

FIG. 5.- A scatter plot showing the velocity residual in the parallel and perpendicular directions for all 53 mock catalogs. The blue dots are in real space and the red crosses are in redshift space, while black dots show recovery from the full dark matter density field in real space. The rms values of the parallel and perpendicular residuals are listed in the left and right panels, respectively. Top and bottom panels correspond to velocity reconstruction with $R_{\text {out }}=250 h^{-1} \mathrm{Mpc}$ and $R_{\text {out }}=100 h^{-1} \mathrm{Mpc}$, respectively. The rms of the parallel and perpendicular residuals are indicated, respectively, in the left and right panels.

velocity residuals vs. the true velocity $\boldsymbol{v}_{\mathrm{lg}}^{\mathrm{tru}}$ (left panels) and vs. the perpendicular component of the reconstructed velocity, $v_{\mathrm{lg}, \perp}^{\mathrm{rec}}$ (right panels) in each of the 53 mocks, The filled black dots refer to the case of dark matter particles reconstruction in real space. Open blue dots and red crosses refer to reconstructions with mock galaxies in real and redshift space, respectively. In the upper panels the reconstructed quantities are measured at $R_{\text {out }}=250 h^{-1} \mathrm{Mpc}$, to match the simulation size. This represents the ideal and rather unrealistic case of a deep, all-sky survey with a selection function accurately estimated out to very large distances. The bottom panel, in which $R_{\text {out }}=100 h^{-1} \mathrm{Mpc}$, represents a more realistic case in which, like in the 2MRS, the errors in the selection function are reasonably small out to $\sim 100 h^{-1} \mathrm{Mpc}$ (Branchini et al. 2012).

The fact that in all plots the mean of the parallel residuals is zero is just a consequence of the choice of the $\beta$ value used in the reconstructions. Instead, the fact that the parallel residuals are uncorrelated with $\boldsymbol{v}_{\mathrm{lg}}^{\mathrm{tru}}$ but anti-correlated with $v_{\mathrm{lg}, \perp}^{\mathrm{rec}}$ is a genuine result. The anticorrelation implies some degeneracy between the error in the estimation of the direction and amplitude in the reconstruction of the LG motion and it should be kept in mind in realistic applications. The accuracy of the LG velocity reconstruction is quantified by the $r m s$ of the parallel residuals and is indicated in the plot. As expected it is smallest when the reconstruction is performed in real space with dark matter particles and larger when one considers mock galaxies at their redshift space positions. Moreover, the scatter for $R_{\text {out }}=100 h^{-1} \mathrm{Mpc}$ is about twice as large as in the respective reconstructions for $R_{\text {out }}=250 h^{-1} \mathrm{Mpc}$. In the realistic case in which the reconstruction is performed in redshift space using all mock 2MRS galaxies within $100 h^{-1} \mathrm{Mpc}$ the rms scatter is of the order of $272 \mathrm{~km} \mathrm{~s}^{-1}$, a value that represents the typical error on the estimated LG motion from currently available all-sky surveys.

The scatter plot in Figure (5) can help to investigate in detail the error budget of the LG velocity reconstruction. Let us consider the case of $R_{\text {out }}=250 h^{-1} \mathrm{Mpc}$. For LG velocity reconstructions with mock galaxies in real space the rms of the total residual, i.e. is the sum in quadrature of the $r m s$ values of the parallel and perpendicular residuals, is $127 \mathrm{~km} \mathrm{~s}^{-1}$. This is higher that the $r m s$ value of $93 \mathrm{~km} \mathrm{~s}^{-1}$ obtained using dark matter particles. Part of the difference is accounted for by shot noise which provide an additional contribution of $\sim 70 \mathrm{~km} \mathrm{~s}^{-1}$, as shown in 55.1 .1 . We attribute the remaining, small discrepancy, to the stochastic nature of the biasing relation seen in figure (1). In redshift space the total scatter of the residuals is about $20 \%$ higher than in real space. This additional uncertainty must be due to non-linear effects which leak differently in real and redshift space and to the multivalued nature of the real-to-redshift space mapping in regions of high density.

\section{THE "KAISER ROCKET" EFFECT}

Redshift surveys are characterized by different types of selection effects that may depend on the intrinsic properties of the objects and the distances. These effects are quantified by a selection function, $\varphi$. Let us focus on the distance dependence and consider the case of a flux limited survey, like 2MRS. Galaxies must be weighted by the inverse of the selection function to compensate for the unobserved faint galaxies with fluxes falling below the detection limit. The weight assigned to a galaxy should be proportional to $\varphi^{-1}(r)$ evaluated at the unknown distance to the galaxy, whereas in practice the selection function is measured at the redshift of the galaxies. All tests used above have indeed used $\varphi^{-1}(r)$. The use of $\varphi^{-1}(s)$, the selection function evaluated at the redshift of the galaxy, leads to systematic biases in the reconstruction of the LG motion and bulk flows: the so-called "Kaiser rocket" effect (Kaiser 1987). In order to demonstrate the importance of the effect we have recovered the LG motion with galaxies weighted by $\varphi^{-1}(s)$ rather than $\varphi^{-1}(r)$. The corresponding residuals are shown as black curves in figure (6). In a survey like $2 \mathrm{MRS}$ the "Kaiser rocket" effect remains tamed for $R_{\text {out }} \lesssim 100 h^{-1} \mathrm{Mpc}$, but increases substantially at larger distances, overshooting to large values as $R_{\text {out }}$ approaches $250 h^{-1} \mathrm{Mpc}$. This behavior explains why we have presented results for $R_{\text {out }}=100 h^{-1} \mathrm{Mpc}$ in figure (5) as the appropriate value for 2MRS-like surveys.

There are several ways one could attempt a correction for this effect. To linear order, this introduces a correc- 
tion term to the general linear theory relation in redshift space (Nusser \& Davis 1994), which could be solved directly using standard numerical method. This will yield an estimate of the velocity field, $\boldsymbol{v}$, of galaxies in the LG frame, from which the reflex dipole at large distances could be computed and identified with the negative of LG motion with respect to the CMB, as explained in the Appendix. Another strategy is to adopt an iterative approach. At the iteration step $i$ the selection function is computed at $\tilde{r}=s-\tilde{r} \hat{\boldsymbol{s}} \cdot \boldsymbol{v}^{i-1} / H_{0}$ where $\boldsymbol{v}^{i-1}$ is the reflex dipole motion of a shell obtained from the previous iteration, starting with zero at the first iteration. In these iterations, the reflex dipole approximates the velocity field of galaxies at the same redshift. This is rigorously correct to linear order where the Kaiser effect does not mix different multipoles of the velocity field, leaving the dipole as the only relevant component for the recovery of the LG motion. This iterative scheme allows the use of the new relation (equation 6) derived here.

In practice, these iterations are time consuming and to assess the impact of the effect in this Section we simply use $\varphi(\tilde{r})$ with $\tilde{r}$ given from the reflex dipole (equation A8 recovered from linear theory using the correct selection function $\varphi(r)$ evaluated at the actual galaxy distance, $r$. The residuals in the reconstruction including this correction are plotted as the red curves in figure (6). The correction manages to suppress the overshooting at large distances and brings the reconstruction closer to corresponding curves in figure (4). But the total rms scatter is still significant- $200 \mathrm{~km} \mathrm{~s}^{-1}$ at $R_{\text {out }}=250 h^{-1} \mathrm{Mpc}$. We emphasize that this is an oversimplified test of the correction to the Kaiser effect. For real catalogs, the determination of the selection function invokes an assessment of galaxy evolution that might be significant even within $R \gtrsim 100 h^{-1}$ Mpc. Further, the short cut we have taken to derive the distance $\tilde{r}$ assumes that the final outcome of the iteration procedure is as accurate as using the reconstruction with $\phi(r)$. Had this been true, the red curves in figure (6) would have coincided with the corresponding curves in figure (4), which is not the case. Therefore, the correction to Kaiser in realistic application is far more uncertain.

\section{DISCUSSION}

In this paper we have investigated the various error sources in the determination of the LG motion.

- It is a mistake to do the analysis in the CMB frame because this frame is only gradually reached. The LG frame gives a better estimate of the distance to the nearby mass fluctuations, as seen in the substantial deviations in the residual vector differences for $R_{\text {out }}<70 h^{-1} \mathrm{Mpc}$ (e.g. figure (3p).

- By far the main source of error is related to the limited depth of galaxy surveys, i.e. cosmic variance. The error in the LG motion estimated from the full dark matter density field in real space and within a radius of $250 h^{-1} \mathrm{Mpc}$ is $\sim 90 \mathrm{~km} \mathrm{~s}^{-1}$. However, the dilute sampling and flux-limited nature of most avalable datasets makes them significantly shallower than this. For an all sky survey like the 2MRS, the contribution to the LG motion can be assessed reliably only within $\sim 100 h^{-1}$ Mpc. At

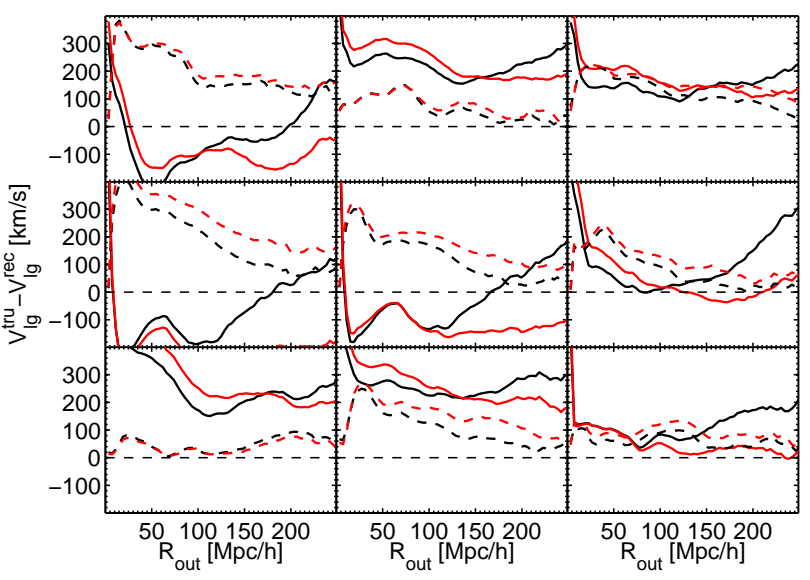

FIG. 6. - Illustration of the rocket effect in the recovery of LG motion. Black curves are residuals from redshift space reconstructions with galaxies weighted by the selection function evaluated at the redshift space coordinate rather the actual distance. Red curves are result of correcting for the rocket effect, as described in the text, in the recovery of the LG motion. Solid and dashed curves correspond to parallel and perpendicular residuals.

this depth, the error in the predicted LG motion is $\sim 200 \mathrm{~km} \mathrm{~s}^{-1}$.

- The velocity residuals measured in the perpendicular and parallel directions allow us to estimate the rms angle of the LG velocity from the full sky data. We find that errors of $\approx 10^{\circ}$ are inevitable, and are not sensitive to whether the analysis is done in redshift space or real space.

- Another source of uncertainty are the errors in the dynamical reconstruction. To assess their contribution to the total error budget we have estimated the LG motion from the full dark matter out to the largest possible outer radius, i.e. $250 h^{-1} \mathrm{Mpc}$. Then, we have filtered out the contribution from large scale structure beyond this radius by measuring the LG motion with respect to the bulk flow of the sphere. Hence the resultant errors are entirely due to dynamical inaccuracies of linear theory. The corresponding $1 \sigma$ error, $\sim 90 \mathrm{~km} \mathrm{~s}^{-1}$, is substantially smaller that the typical error in linear reconstruction of the peculiar velocity of a generic observer in the Universe. The reason for this is the strict criteria we have applied in selecting the "LG observer" in the mock catalogs, aimed at matching the quietness and moderate density environment of the observed LG. Removing these selection criteria boosts the error to $\gtrsim 300 \mathrm{~km} \mathrm{~s}^{-1}$, consistent with previous studies (Nusser \& Branchini 2000).

Nonlinear dynamical reconstruction methods (e.g. Shaya et al. 1995; Croft \& Gaztanaga 1997; Frisch et al. 2002, Nusser \& Branchini 2000; Branchini et al. 2002) can potentially reduce the dynamical error. However, because the particular environment of the LG, errors due to linear reconstruction are subdominant compared to the total error budget discussed above.

- The shot noise originating from the sampling of the mass density field by a finite number of tracers, forcing the use of the selection function of the 
sample $\varphi$ to deal with the magnitude limit, is another error source. In the case of the 2MRS galaxies brighter than $\mathrm{K}_{\mathrm{s}}=11.75$, the error amplitude is comparable to that of the dynamical error. The rms of the combined dynamical and shot noise scatter is close to the error estimated directly from the scatter of the LG motions reconstructed from our mock galaxy catalogs, leaving little room for any substantial, additional source of error.

- Indeed, we have identified the remaining error source with galaxy biasing. Galaxies in our mocks follow a biasing relation that is close to linear in regions with positive densities but it significantly deviates from linearity in voids where galaxies are less abundant than expected from an extrapolation of the linear bias. Moreover, the biasing relation is non-deterministic. Its scatter is driven by Poisson noise everywhere but in high density regions, where the intrinsic scatter in the biasing relation is dominant. Nonetheless, uncertainties due to deviations from the assumptions of linear biasing are of relative insignificance as illustrated by the comparison of the real space reconstructions from 2MRS-like mocks generated from dark matter particles and the mock galaxy catalogs.

- Reconstructing velocities from redshift space data is fundamentally a more challenging problem than in real space. Effects like multi-valued zones (tracers with distinct distances along the same line of sight, but with very similar redshifts) and fingers of god (spreading of galaxies in virialized regions along the line of sight) affect the reconstruction on scales larger than the traditional non-linear scale. In order to mitigate the effect of the fingers of god, the redshifts of the mock galaxies have been computed with peculiar velocities smoothed on a scale $1 h^{-1} \mathrm{Mpc}$ scale. The $1 \sigma$ errors in the LG motion reconstructed in redshift space reconstruction is $\sim 20 \%$ larger than in real space.

- We have also assessed the "Kaiser rocket" effect and demonstrated that it can be partially corrected if the selection function is well constrained by observations. Correction is easy in the case of mock catalogs but more challenging in real datasets where the effect of galaxy evolution cannot be ignored, even within the local volume encompassed by the 2MRS (Branchini et al. 2012). A distinct signature of the "Kaiser rocket" effect is the overshooting of the reconstructed LG motion at large radii, ( $\gtrsim 100 h^{-1} \mathrm{Mpc}$ ). We are not aware of any reconstruction of galaxy dipoles from real datasets taking into account this spurious growth.

Despite the general consensus on its origin, the identification of the actual gravitational sources responsible for the LG motion is still a matter of debate. Gravity is a long range force and it may prove futile to try to identity specific sources for the LG motion. A more useful description is in terms of "dipole convergence scale", i.e. the physical distance which encompasses the matter fluctuations responsible for generating most of the LG motion. In the standard $\Lambda \mathrm{CDM}$ model, the dominant contribution to the LG motion is expected to arise from mass fluctuations with a distance up to $\sim 200-300 h^{-1} \mathrm{Mpc}$ from the LG (Bilicki et al. 2011). We have seen that convergence in the mocks is achieved at a depth of $\approx 250 h^{-1} \mathrm{Mpc}$, consistent with the theoretical expectation. The convergence is gradual, indicating that the cumulative effect of the large scale mass density field must be invoked to account for the LG motion,

The $150-200 \mathrm{~km} \mathrm{~s}^{-1}$ accuracy should be regarded as a conservative estimate for the expected error in the estimates of the LG velocity. Indeed, comparisons between the observed LG motion and the prediction from various redshift surveys have yielded consistency up to the level we see here in the mocks. We, therefore, conclude that the standard picture for the formation of large scale structure is fully consistent with current observational data of the large scale motions.

\section{ACKNOWLEDGMENT}

Special thanks are due to Gerard Lemson for providing us with data from the German Astrophysical Observatory (GAVO). This research was supported by the I-CORE Program of the Planning and Budgeting Committee, THE ISRAEL SCIENCE FOUNDATION (grants No. 1829/12 and No. 203/09), the German-Israeli Foundation for Research and Development, and the Asher Space Research Institute. EB acknowledges the financial support provided by MIUR PRIN 2011 'The dark Universe and the cosmic evolution of baryons: from current surveys to Euclid' and Agenzia Spaziale Italiana for financial support from the agreement ASI/INAF/I/023/12/0. AN and EB Thanks the Department of Astronomy at the University of Cape Town for hospitality. MD acknowledges funding from CAASTRO/Swinburne where he completed this work.

\section{REFERENCES}

Basilakos, S., \& Plionis, M. 2006, MNRAS, 373, 1112

Benson, A. J., Cole, S., Frenk, C. S., Baugh, C. M., \& Lacey, C. G. 2000, MNRAS, 311, 793

Bilicki, M., Chodorowski, M., Jarrett, T., \& Mamon, G. A. 2011, ApJ, 741, 31

Branchini, E., Davis, M., \& Nusser, A. 2012, MNRAS, 424, 472

Branchini, E., Eldar, A., \& Nusser, A. 2002, MNRAS, 335, 53

Branchini, E., Plionis, M., \& Sciama, D. W. 1996, ApJL, 461, L17+

Chodorowski, M. J., \& Ciecielag, P. 2002, MNRAS, 331, 133

Chodorowski, M. J., Coiffard, J.-B., Bilicki, M., Colombi, S., \& Ciecielag, P. 2008, MNRAS, 389, 717

Ciecielg, P., \& Chodorowski, M. J.' 2004, MNRAS, 349, 945

Coles, P. 1993, MNRAS, 262, 1065

Coles, P., Melott, A. L., \& Munshi, D. 1999, ApJL, 521, L5
Courteau, S., \& van den Bergh, S. 1999, AJ, 118, 337

Croft, R. A. C., \& Gaztanaga, E. 1997, MNRAS, 285, 793

Dale, D. A., Giovanelli, R., Haynes, M. P., Campusano, L. E., Hardy, E., \& Borgani, S. 1999, ApJL, 510, L11

Davis, M., \& Huchra, J. 1982, ApJ, 254, 437

Davis, M., Nusser, A., Masters, K. L., Springob, C., Huchra, J. P., \& Lemson, G. 2011, MNRAS, 413, 2906

Davis, M., Nusser, A., \& Willick, J. A. 1996, ApJ, 473, 22

Davis, M., Strauss, M. A., \& Yahil, A. 1991, ApJ, 372, 394

De Lucia, G., \& Blaizot, J. 2007, MNRAS, 375, 2

D'Mellow, K. J., Saunders, W., \& PSCz/BTP Teams. 2004, PASA, 21, 415

Fixsen, D. J., Cheng, E. S., Gales, J. M., Mather, J. C., Shafer, R. A., \& Wright, E. L. 1996, ApJ, 473, 576 
Frisch, U., Matarrese, S., Mohayaee, R., \& Sobolevski, A. 2002, Nature, 417, 260

Fry, J. N., \& Gaztanaga, E. 1993, ApJ, 413, 447

Gramann, M. 1993, ApJ, 405, 449

Harmon, R. T., Lahav, O., \& Meurs, E. J. A. 1987, MNRAS, 228 $5 \mathrm{P}$

Hinshaw, G., Weiland, J. L., Hill, R. S., Odegard, N., Larson, D. Bennett, C. L., Dunkley, J., Gold, B., Greason, M. R., Jarosik, N., Komatsu, E., Nolta, M. R., Page, L., Spergel, D. N., Wollack, E., Halpern, M., Kogut, A., Limon, M., Meyer, S. S., Tucker, G. S., \& Wright, E. L. 2009, ApJ. S, 180, 225

Huchra, J. P., Macri, L. M., Masters, K. L., Jarrett, T. H., Berlind, P., Calkins, M., Crook, A. C., Cutri, R., Erdoğdu, P., Falco, E., George, T., Hutcheson, C. M., Lahav, O., Mader, J., Mink, J. D., Martimbeau, N., Schneider, S., Skrutskie, M., Tokarz, S., \& Westover, M. 2012, ApJ. S, 199, 26

Hudson, M. J. 1993, MNRAS, 265, 72

Huff, E., Schulz, A. E., White, M., Schlegel, D. J., \& Warren, M. S. 2007, Astroparticle Physics, 26, 351

Humason, M. L., Mayall, N. U., \& Sandage, A. R. 1956, AJ, 61, 97

Juszkiewicz, R., Vittorio, N., \& Wyse, R. F. G. 1990, ApJ, 349, 408

Kaiser, N. 1987, MNRAS, 227, 1

-. 1988, MNRAS, 231, 149

Karachentsev, I. D., \& Makarov, D. A. 1996, AJ, 111, 794

Kauffmann, G., Nusser, A., \& Steinmetz, M. 1997, MNRAS, 286, 795

Kirshner, R. P., Oemler, Jr., A., \& Schechter, P. L. 1979, AJ, 84, 951

Kocevski, D. D., \& Ebeling, H. 2006, ApJ, 645, 1043

Kogut, A., Lineweaver, C., Smoot, G. F., Bennett, C. L., Banday, A., Boggess, N. W., Cheng, E. S., de Amici, G., Fixsen, D. J., Hinshaw, G., Jackson, P. D., Janssen, M., Keegstra, P., Loewenstein, K., Lubin, P., Mather, J. C., Tenorio, L., Weiss, R., Wilkinson, D. T., \& Wright, E. L. 1993, ApJ, 419, 1

Lahav, O. 1987, MNRAS, 225, 213

Lahav, O., Lynden-Bell, D., \& Rowan-Robinson, M. 1988, MNRAS, 234, 677

Linder, E. V. 2005, Physical Review D., 72, 043529

Lynden-Bell, D. 1981, The Observatory, 101, 111

Lynden-Bell, D., Lahav, O., \& Burstein, D. 1989, MNRAS, 241 325

Meiksin, A., \& Davis, M. 1986, AJ, 91, 191

Narayanan, V. K., Berlind, A. A., \& Weinberg, D. H. 2000, ApJ, 528,1

Nusser, A., \& Branchini, E. 2000, MNRAS, 313, 587

Nusser, A., \& Colberg, J. M. 1998, MNRAS, 294, 457

Nusser, A., \& Davis, M. 1994, ApJL, 421, L1

Nusser, A., Dekel, A., Bertschinger, E., \& Blumenthal, G. R. 1991, ApJ, 379, 6

Peebles, P. J. E. 1980, The large-scale structure of the universe (Princeton University Press)

Planck Collaboration, Aghanim, N., Armitage-Caplan, C., Arnaud, M., Ashdown, M., Atrio-Barandela, F., Aumont, J., Banday, A. J., Barreiro, R. B., Bartlett, J. G., Benabed, K., Benoit-Lévy, A., \& Bernard, J.-P. 2013, ArXiv e-prints
Plionis, M. 1988, MNRAS, 234, 401

Plionis, M., \& Kolokotronis, V. 1998, ApJ, 500, 1

Plionis, M., \& Valdarnini, R. 1991, MNRAS, 249, 46

Rauzy, S., \& Gurzadyan, V. G. 1998, MNRAS, 298, 114

Rowan-Robinson, M., Sharpe, J., Oliver, S. J., Keeble, O. Canavezes, A., Saunders, W., Taylor, A. N., Valentine, H., Frenk, C. S., Efstathiou, G. P., McMahon, R. G., White, S. D. M., Sutherland, W., Tadros, H., \& Maddox, S. 2000 , MNRAS, 314, 375

Sandage, A. 1986, ApJ, 307, 1

Scaramella, R., Vettolani, G., \& Zamorani, G. 1991, ApJL, 376, L1

-. 1994, ApJ, 422, 1

Scherrer, R. J., \& Weinberg, D. H. 1998, ApJ, 504, 607

Schmoldt, I., Branchini, E., Teodoro, L., Efstathiou, G., Frenk, C. S., Keeble, O., McMahon, R., Maddox, S., Oliver, S., Rowan-Robinson, M., Saunders, W., Sutherland, W., Tadros, H., \& White, S. D. M. 1999a, MNRAS, 304, 893

Schmoldt, I. M., Saar, V., Saha, P., Branchini, E., Efstathiou, G. P., Frenk, C. S., Keeble, O., Maddox, S., McMahon, R. Oliver, S., Rowan-Robinson, M., Saunders, W., Sutherland, W. J., Tadros, H., \& White, S. D. M. 1999b, AJ, 118, 1146 Seljak, U. 2001, MNRAS, 325, 1359

Shaya, E. J., Peebles, P. J. E., \& Tully, R. B. 1995, ApJ, 454, 15

Smith, R. E., Scoccimarro, R., \& Sheth, R. K. 2007, Physical Review D., 75, 063512

Springel, V., White, S. D. M., Jenkins, A., Frenk, C. S., Yoshida, N., Gao, L., Navarro, J., Thacker, R., Croton, D., Helly, J., Peacock, J. A., Cole, S., Thomas, P., Couchman, H., Evrard, A., Colberg, J., \& Pearce, F. 2005, Nature, 435, 629

Strauss, M. A., Yahil, A., Davis, M., Huchra, J. P., \& Fisher, K. 1992, ApJ, 397, 395

Tegmark, M., Zaldarriaga, M., \& Hamilton, A. J. 2001, Physical Review D., 63, 043007

Turner, E. L. 1979, ApJ, 231, 645

van den Bergh, S. 2000, Cambridge Astrophysics Series, 35

Verde, L., Heavens, A. F., Percival, W. J., Matarrese, S., Baugh, C. M., Bland-Hawthorn, J., Bridges, T., Cannon, R., Cole, S., Colless, M., Collins, C., Couch, W., Dalton, G., De Propris, R., Driver, S. P., Efstathiou, G., Ellis, R. S., Frenk, C. S.,

Glazebrook, K., Jackson, C., Lahav, O., Lewis, I., Lumsden, S. Maddox, S., Madgwick, D., Norberg, P., Peacock, J. A. Peterson, B. A., Sutherland, W., \& Taylor, K. 2002, MNRAS, 335,432

Villumsen, J. V., \& Strauss, M. A. 1987, ApJ, 322, 37

Webster, M., Lahav, O., \& Fisher, K. 1997, MNRAS, 287, 425

Westover, M. 2007, PhD thesis, Harvard University

Yahil, A., Sandage, A., \& Tammann, G. A. 1980, ApJ, 242, 448

Yahil, A., Tammann, G. A., \& Sandage, A. 1977, ApJ, 217, 903

Yahil, A., Walker, D., \& Rowan-Robinson, M. 1986, ApJL, 301, L1

Zaroubi, S., Hoffman, Y., \& Dekel, A. 1999, ApJ, 520, 413

APPENDIX

\section{A. RECONSTRUCTING LG MOTION}

\section{A.1. Relations for continuous fields}

The LG motion is a special case of the bulk flow. Thus we first derive a linear relation between the bulk flow and the density contrast $\delta(\boldsymbol{r})=\rho(\boldsymbol{r}) / \bar{\rho}-1$. The bulk flow of a sphere of radius $R$ centered at the origin

$$
\mathbf{B}=\frac{1}{V} \int_{r<R} \mathrm{~d} r^{3} \boldsymbol{v}(\boldsymbol{r})
$$

where $V=4 \pi / 3 R^{3}$.

We note the mathematical identity ${ }^{1} \int_{V} \boldsymbol{\nabla} g \mathrm{~d}^{3} r=\int_{S} g \mathrm{~d} \boldsymbol{S}$ where $g$ is a scalar function, the vector $\mathrm{d} \boldsymbol{S}$ is an element of the surface enclosing the volume $V$. Applying this identity with $g=-\phi$ and noting the definition of $\mathbf{B}$ in A1, yields

$$
\begin{aligned}
\mathbf{B} & =-\frac{1}{V} \int_{S} \phi \mathrm{d} \boldsymbol{S} \\
& =-\frac{1}{V} \int \phi(|\boldsymbol{s}|=r, \hat{\boldsymbol{s}}) \hat{\boldsymbol{s}} r^{2} \mathrm{~d} \Omega,
\end{aligned}
$$

1 This is a particular form of the Gauss (or Green) Theorem $\int_{V} \boldsymbol{\nabla} \cdot \boldsymbol{A} \mathrm{d}^{3} r=\int \boldsymbol{A} \cdot \mathrm{d} \boldsymbol{S}$ when one consider a scalar field $\boldsymbol{A}=A$ and

$\nabla \cdot=\nabla$ 
where the second line is valid for a spherical surface of radius $R$ centered on the origin. We substitute $\phi$ in terms of spherical harmonics expansion over $\phi_{l m}$ from $\left[4\right.$. Thanks to the orthogonality condition $\int Y_{l m} Y_{l^{\prime} m^{\prime}}^{*} \mathrm{~d} \Omega=\delta_{l l^{\prime}}^{K} \delta_{m m^{\prime}}^{K}$, only $l=1$ contributes to the integral, with the net result,

$$
\begin{aligned}
\mathbf{B} & =-\frac{H_{0} \beta /(1+\beta)}{2 \tilde{l}+1} V^{-1}\left[r^{\tilde{l}+2} \int_{0}^{r} \frac{\boldsymbol{\Delta}(u)}{u^{\tilde{l}-1}} \mathrm{~d} u\right. \\
& \left.-r^{1-\tilde{l}} \int_{0}^{r} \boldsymbol{\Delta}(u) u^{\tilde{l}+2} \mathrm{~d} u\right],
\end{aligned}
$$

where $\Delta_{z}=\sqrt{4 \pi / 3} \delta_{10}^{\mathrm{s}}, \Delta_{y}=i \sqrt{2 \pi / 3}\left(\delta_{11}^{\mathrm{s}}+\delta_{1-1}^{\mathrm{s}}\right), \Delta_{x}=-\sqrt{2 \pi / 3}\left(\delta_{11}^{\mathrm{s}}-\delta_{1-1}^{\mathrm{s}}\right)$ and $(1+\beta) \tilde{l}(\tilde{l}+1)-2=0$ since $l=1$. In the derivation we have also used the relations $\hat{\boldsymbol{z}} \cdot \hat{\boldsymbol{r}}=\cos \theta=\sqrt{4 \pi / 3} Y_{10}, \hat{\boldsymbol{y}} \cdot \hat{\boldsymbol{r}}=\sin \theta \sin \varphi=i \sqrt{2 \pi / 3}\left(Y_{11}+Y_{1-1}\right)$ and $\hat{\boldsymbol{x}} \cdot \hat{\boldsymbol{r}}=\sin \theta \cos \varphi=-\sqrt{2 \pi / 3}\left(Y_{11}-Y_{1-1}\right)$.

The bulk motion of a thin shell, i.e. the reflex dipole motion of the shell, is defined as

$$
\boldsymbol{v}^{\text {shell }}(r)=\frac{1}{4 \pi} \int \boldsymbol{v}(\boldsymbol{r}) \mathrm{d} \Omega .
$$

Thus

$$
\boldsymbol{v}^{\text {shell }}=\frac{1}{3 R^{2}} \frac{\partial\left[R^{3} \mathbf{B}(R)\right]}{\partial R}
$$

For completeness, we also note that an application of the mathematical identity above to compute the mean motion of a shell of radius $r$ and thickness $\delta r \rightarrow 0$, yields

$$
\boldsymbol{v}^{\text {shell }}=-\frac{1}{4 \pi} \int\left[\frac{\mathrm{d} \phi}{\mathrm{d} r}+2 \frac{\phi}{r}\right] \mathrm{d} \Omega .
$$

The LG velocity, $\boldsymbol{v}_{\mathrm{lg}}$, is the negative of the reflex dipole of the shell at infinity so that,

$$
\boldsymbol{v}_{\mathrm{lg}}=-\lim _{r \rightarrow \infty} \boldsymbol{v}^{\text {shell }}(r) \text {. }
$$

Using (A8) and $\mathrm{A} 5$ we get

$$
\begin{aligned}
\boldsymbol{v}^{\text {shell }} & =-\frac{H_{0} \beta /(1+\beta)}{2 \tilde{l}+1}(3 V)^{-1}\left[(\tilde{l}+2) r^{\tilde{l}+2} \int_{0}^{r} \frac{\boldsymbol{\Delta}(u)}{u^{\tilde{l}-1}} \mathrm{~d} u\right. \\
& \left.-(1-\tilde{l}) r^{1-\tilde{l}} \int_{0}^{r} \boldsymbol{\Delta}(u) u^{\tilde{l}+2} \mathrm{~d} u\right] .
\end{aligned}
$$

\section{A.2. Relations for a discrete sampling}

We now modify the above relations for the LG motion and the bulk flow to the case of a discrete sampling of density field in redshift space by a discrete distribution of $N$ tracers, $i=1 \cdots N$, with mean number density $\bar{n}$. This modification will allow an application of linear theory reconstruction directly on the distribution of galaxies in redshift space, rather then employ a smoothing procedure in order to use the relations above. In the discrete representation, the density field is approximated as

$$
\delta(\boldsymbol{s})=\bar{n}^{-1} \sum_{i=1}^{N} s_{i}^{-2} \delta^{D}\left(\boldsymbol{s}-\boldsymbol{s}_{i}\right)
$$

where $\delta^{D}$ is Dirac's delta function and $\boldsymbol{s}_{i}$ are the redshift coordinates of the tracers. If the tracers are galaxies in a flux limited survey, then the contribution of each point should be weighted by a selection function to account for the loss of fainter galaxies at larger distances. For brevity of notation, at this stage we assume a volume limited survey so that all tracers are equally weighted. The spherical harmonics expansion is

$$
\delta_{l m}^{\mathrm{s}}=\bar{n}^{-1} \sum_{i=1}^{N} s_{i}^{-2} \delta^{D}\left(s-s_{i}\right) Y_{l m}\left(\hat{\boldsymbol{s}}_{i}\right),
$$

giving $\boldsymbol{\Delta}=\bar{n}^{-1} \sum_{i} s_{i}^{-2} \delta^{D}\left(s-s_{i}\right) \hat{\boldsymbol{s}}_{i}$. The expression A for the bulk flow becomes

$$
\mathbf{B}(r)=\frac{-r^{\tilde{l}+2}}{(1+\beta)(2 \tilde{l}+1)} \frac{\beta H_{0}}{\bar{n} V} \sum_{s_{i}<r} \frac{\boldsymbol{s}_{i}}{s_{i}^{\tilde{l}+2}}+\frac{r^{1-\tilde{l}}}{(1+\beta)(2 \tilde{l}+1)} \frac{\beta H_{0}}{\bar{n} V} \sum_{s_{i}<r} s_{i}^{\tilde{l}-1} \boldsymbol{s}_{i} .
$$

Another form of this relation which should be more appropriate for numerical applications is

$$
\mathbf{B}(r)=\frac{-\beta H_{0}}{(1+\beta)(2 \tilde{l}+1)}\left[r^{\tilde{l}+2}\left\langle\frac{\boldsymbol{s}_{i}}{s_{i}^{\tilde{l}+2}}\right\rangle_{r}-r^{1-\tilde{l}}\left\langle s_{i}^{\tilde{l}-1} \boldsymbol{s}_{i}\right\rangle_{r}\right] .
$$


where $<X_{i}>_{r}=\sum_{s_{i}<r} X_{i} /(\bar{n} V)$ and is approximated numerically by averaging over particles, i.e. $\bar{n} V$ is approximated as the number of particles within $r$. This form avoids potential problems due to the divergent behavior of $r^{\tilde{l}+2} / V \sim r^{\tilde{l}-1}$ as $r \rightarrow 0$. and

$$
\begin{gathered}
\boldsymbol{v}^{\text {shell }}(r)=\frac{-r^{\tilde{l}-1}}{(1+\beta)} \frac{\beta}{4 \pi \bar{n}} \sum_{s_{i}<r} \frac{\boldsymbol{s}_{i}}{s_{i}^{\tilde{l}+2}}+\frac{1-\tilde{l}}{2 \tilde{l}+1} \frac{r^{-(2+\tilde{l})}}{(1+\beta)} \frac{\beta}{4 \pi \bar{n}} \sum_{s_{i}<r} s_{i}^{\tilde{l}-1} \boldsymbol{s}_{i} . \\
\boldsymbol{v}^{\text {shell }}(r)=\frac{-\beta H_{0}}{(1+\beta)(2 \tilde{l}+1)}\left[\left(\frac{2 \tilde{l}+1}{3}\right) r^{\tilde{l}+2}\left\langle\frac{\boldsymbol{s}_{i}}{s_{i}^{\tilde{l}+2}}\right\rangle_{r}-\left(\frac{1-\tilde{l}}{3}\right) r^{1-\tilde{l}}\left\langle s_{i}^{\tilde{l}-1} \boldsymbol{s}_{i}\right\rangle_{r}\right] .
\end{gathered}
$$

In the relations A8 and A11 it is understood that the sphere of radius $r$ is centered at the origin, $\boldsymbol{r}=0$, of the coordinate system in real space. Since we work in the LG frame, $\boldsymbol{r}=0$ corresponds to $\boldsymbol{s}=0$, hence the sphere is also centered at the origin in redshift space. We note that the contribution of the second term on the r.h.s of A11) becomes increasingly small as $R \rightarrow 0$. This is sustained analytically by the form of this term which gives more weight to larger distances where homogeneity is more pronounced and is corroborated by the analysis of the mock catalogs in Section 5.1. As a consequence the LG velocity can be expressed as

$$
\boldsymbol{v}_{\mathrm{lg}}=\frac{(2 \tilde{l}+1) R_{\mathrm{out}}^{\tilde{l}-1}}{(1+\beta)(2 \tilde{l}+1)} \frac{\beta H_{0}}{4 \pi \bar{n}} \sum_{R_{\mathrm{lg}}<s_{i}<R_{\mathrm{out}}} \frac{\boldsymbol{s}_{i}}{s_{i}^{\tilde{l}+2}}-\frac{(1-\tilde{l}) r^{1-\tilde{l}}}{(1+\beta)(2 \tilde{l}+1)} \frac{\beta H_{0}}{3 \bar{n} V} \sum_{R_{\mathrm{lg}}<s_{i}<R_{\mathrm{out}}} s_{i}^{\tilde{l}-1} \boldsymbol{s}_{i} .
$$

which also appears as equation 6 in the main body of the paper. The sum extend over all objects between $R_{\mathrm{lg}}$, the radius assigned to the $\mathrm{LG}$, and a maximum distance $R_{\text {out }}$. Note that there is no lower cutoff at $R_{\mathrm{lg}}$ in the bulk flow expression A11 simply because the region within $R_{\lg }$ should be counted as part of the sphere $R$ for which the bulk flow is computed. The fact that $R_{\mathrm{lg}} \ll R$ makes the cutoff in A11 insignificant. The maximum distance $R_{\text {out }}$ should be assumed to approach infinity in the ideal case where galaxies are observed over all space. However, in realistic redshift surveys, the number density of galaxies decreases with redshift due to magnitude cuts, making the noise increase dramatically at larger redshifts.

The real space counterparts of the relations A11 and A15 are obtained by setting $\tilde{l}=l=1$ and replacing $f$ with $\beta /(1+\beta)$. This yields

$$
\mathbf{B}=\frac{-H_{0} \beta}{4 \pi \bar{n}} \sum_{r_{i}<R} \frac{\boldsymbol{r}_{i}}{r_{i}^{3}}+\frac{H_{0} \beta}{3 \bar{n} V} \sum_{r_{i}<R} \boldsymbol{r}_{i}
$$

and

$$
\boldsymbol{v}_{\mathrm{lg}}=\frac{H_{0} \beta}{4 \pi \bar{n}} \sum_{R_{\mathrm{out}}>r_{i}>R_{\mathrm{lg}}} \frac{\boldsymbol{r}_{i}}{r_{i}^{3}}
$$

Since $\bar{n} V \approx N$, the total number of objects in the sphere, we see that the second term in the r.h.s. of the bulk flow expression $\mathrm{A} 16$ is proportional to the center of mass coordinate, $\sum \boldsymbol{r}_{i} / N$, as already noticed by Juszkiewicz et al. (1990).

\section{B. THE DEPENDENCE ON $\beta$ IN RECONSTRUCTION FROM REDSHIFT SPACE DATA}

In linear theory, the recovered peculiar velocity field in real space is linearly proportional to $\beta$. It can be shown that nonlinear dynamics preserve this proportionality to a very good approximation (Gramann 1993; Nusser \& Colberg 1998). In redshift space, the non-isotropic enhancement of the density by the radial peculiar velocities introduces a non-trivial dependence on $\beta$. This is evident from equation (6) in which deviations from linearity arise from the explicit dependence of $\tilde{l}$ on $\beta$. We characterize this dependence by a function $F(\beta)=\left|\boldsymbol{v}_{\mathrm{lg}}(\beta)\right| /\left|\boldsymbol{v}_{\mathrm{lg}}\left(\beta=\beta_{0}\right)\right|$ where $\beta_{0}$ is a fixed reference value.

Equation (6) implies that $F$ depends also on the actual distribution of tracers. Hence, there is no universal form for $F(\beta)$ which is valid for any distribution. Here we focus on the $2 \mathrm{MRS}$ and explore the dependence on $\beta$ from the mocks. We proceed as follows. For each mock we perform the sum in equation 6 over all galaxies within $R_{\text {out }}=250 h^{-1} \mathrm{Mpc}$, for several values of $\beta$. For each $\beta$, we then compute the mean and standard deviation of $F(\beta)=\left|\boldsymbol{v}_{\mathrm{lg}}(\beta)\right| / \mid \boldsymbol{v}_{\mathrm{lg}}(\beta=0.3)$ from all the mocks. The black dots in figure $(7)$ showing the mean $\langle F(\beta)\rangle$, significantly deviates from the linear scaling (dashed line) appropriate for real space reconstruction. The standard deviation is represented by the error-bars and it reflects the scatter in $F(\beta)$ due to variations of the galaxy distributions among the mocks. The blue, solid curve represents a polynomial fit to the black dots,

$$
<F>\approx \frac{F_{0} \beta}{\left(1+7 \beta^{3 / 2}\right)^{2 / 3}},
$$

where $F_{0}$ is a constant such that $F(\beta=0.3)=1$. 


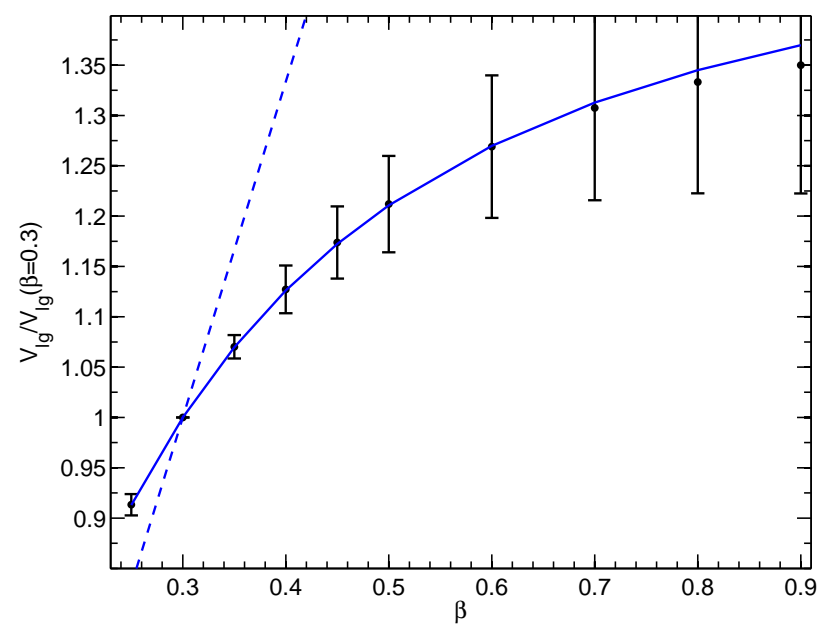

FIG. 7.- The dependence of the redshift space reconstruction of $\boldsymbol{v}_{\mathrm{lg}}$ on $\beta$. The black dots are the average, from all 53 mock, of $\left|\boldsymbol{v}_{\mathrm{lg}}\right|$ scaled by its value at $\beta=0.3$ and the error-bars are the corresponding $r m s$ scatter. The blue solid curve is a fit, $F_{0} \beta /\left(1+7 \beta^{3 / 2}\right)^{2 / 3}$, to the the black dots. The dashed line is the dependence of real space reconstruction on $\beta$, i.e. $(\beta / 0.3)$. 